\title{
Validación cualitativa y cuantitativa de un instrumento para medir la satisfacción estudiantil universitaria
}

\author{
Qualitative and quantitative validation of an instrument \\ to measure university student satisfaction
}

Validação qualitativa e quantitativa de um instrumento
para medir a satisfação do estudante com a educação universitária

Recibido - Received - Recibido: 10 / 06 / 2021 Corregido - Revised - Revisado: 09 / 09 / 2021 Aceptado - Accepted - Aprobado: 225 / 10 / 2021

DOI: https://doi.org/10.22458/ie.v23i35.3590

URL: https://revistas.uned.ac.cr/index.php/innovaciones/article/view/3590

\begin{abstract}
Resumen: El objetivo principal de la presente investigación es validar cualitativa y cuantitativamente el instrumento para medir la satisfacción de los estudiantes universitarios con su educación (SEUE), el cual fue desarrollado por Gento y Vivas (2003), y, se empleará posteriormente para evaluar la percepción del alumnado de Ingeniería de La Universidad del Zulia, con el fin de determinar las relaciones existentes entre la satisfacción con respecto a las variables medidas y su desempeño académico. Para lograr este objetivo, se sometió a juicio de expertos el instrumento previamente diseñado para determinar su validez cualitativa, y así realizar las modificaciones necesarias, para luego aplicarlo a una muestra probabilística compuesta de 336 sujetos de una población total de 2653 estudiantes activos de la Facultad de Ingeniería de la Universidad del Zulia. Con estos datos, se calculó la confiabilidad del instrumento empleando el Alfa de Cronbach y se procedió a realizar la prueba de correlación de Pearson. Posteriormente, se efectúo un análisis factorial exploratorio para determinar la validez del constructo y lograr reducir las dimensiones diseñadas inicialmente, para luego calcular la validez de criterio (validez concurrente) empleando un instrumento patrón. Finalmente, se determinó la estabilidad y el rendimiento del instrumento. Al finalizar, se pudo concluir que las dimensiones podían ser reducidas a seis y la Satisfacción Estudiantil Universitaria podía ser medida por medio de este instrumento; ya que demostró poseer estabilidad y repoducibilidad para ser empleado en futuras investigaciones.
\end{abstract}

Palabras Clave: método de medición, instrumento de medición, satisfacción, estudiante universitario.

Summary: The main objective of this research is to validate qualitatively and quantitatively the instrument to measure the satisfaction of university students with their education (SEUE). This instrument was developed by Gento and Vivas (2003), and will be used later to evaluate the perception of Engineering students of the University of Zulia, in order to determine the relationships between satisfaction with respect to the variables measured and their academic performance. To achieve this objective, the previously designed instrument was submitted to expert judgment to determine its qualitative validity, and thus make the necessary modifications, to then apply it to a probabilistic sample composed of 336 subjects from a total population of 2653 active students of the School of Engineering of the University of Zulia. With these data, the reliability of the instrument was calculated using Cronbach's Alpha and the Pearson correlation test was carried out. Subsequently, an exploratory factor analysis was carried out to determine the validity of the construct and to reduce the dimensions initially designed, to later calculate the criterion validity (concurrent validity) using a standard instrument. Finally, the stability and performance of the instrument was determined. At the end, it was possible to conclude that the dimensions could be reduced to six and the University Student Satisfaction could be measured by means of this instrument, since it proved it possesses stability and reproducibility to be used in future research.

Key Words: measurement method, measuring instrument, satisfaction, college student. 
Resumo: $O$ principal objetivo desta pesquisa é validar qualitativa e quantitativamente o instrumento para medir a satisfação dos estudantes universitários com sua educação (SEUE), que foi desenvolvido por Gento e Vivas (2003), e que posteriormente será utilizado para avaliar a percepção dos estudantes de Engenharia da Universidade de Zulia, para determinar as relações existentes entre a satisfação com relação às variáveis medidas e seu desempenho acadêmico. Para alcançar este objetivo, o instrumento previamente projetado foi submetido ao julgamento de especialistas para determinar sua validade qualitativa, e assim fazer as modificações necessárias, para depois ser aplicado a uma amostra de probabilidade de 336 sujeitos de uma população total de 2653 estudantes ativos na Faculdade de Engenharia da Universidad del Zulia. Com estes dados, a confiabilidade do instrumento foi calculada usando o alfa de Cronbach para fazer o teste de correlação de Pearson. Posteriormente, foi realizada uma análise de fatores exploratórios para determinar a validade do construto para reduzir as dimensões inicialmente projetadas, a fim de então calcular a validade do critério (validade concorrente) usando um instrumento padrão. Finalmente, foi determinada a estabilidade e o desempenho do instrumento. No final, pode-se concluir que as dimensões poderiam ser reduzidas a seis e a Satisfação do Estudante Universitário poderia ser medida por meio deste instrumento; uma vez que ele provou possuir estabilidade e reprodutibilidade para ser empregado em pesquisas futuras.

Palavras-chave: método de medição, instrumento de medição, satisfação, estudante universitário.

\section{INTRODUCCIÓN}

La satisfacción estudiantil, la cual Surdez (2018) define como "el bienestar que experimentan los alumnos por sentir cubiertas sus expectativas académicas como resultado de las actividades que realiza la institución para atender sus necesidades educativas" (p. 4), es un aspecto que debe ser de primordial atención en cualquier entidad educativa; pues refleja los procesos que se llevan a cabo dentro de ellas.

En el caso de las universidades, la satisfacción es un elemento que puede influenciar no solo la actitud de los estudiantes ante las condiciones a las que se enfrentan, sino que algunos investigadores, como Lizzio y colaboradores (2002) plantean que una percepción positiva del ambiente de estudio puede tener una relación directa con el desempeño de los mismos (p.17), y algunos otros, como de Vries (2011) tuvieron hallazgos un poco más alarmantes; pues concluyen que los estudiantes universitarios que se muestran insatisfechos son más propensos a abandonar sus estudios (p. 15), lo cual puede traer consecuencias alarmantes, no solo para el individuo, sino para la sociedad, aparte del alto costo económico que representa para el Estado y las familias y la sociedad. (Baez, 2013: 7)

Adicionalmente, la medición del nivel de satisfacción estudiantil muchos investigadores lo consideran como un indicador para la gestión de la calidad de la educación, como lo plantean Sandoval et al. (2016), quienes exponen que el estudio de la Satisfacción Estudiantil debe ser considerado en las Instituciones de Educación Superior, debido a que el grado de satisfacción de los estudiantes refleja la eficacia en la calidad de la educación que se imparte (p. 705), lo cual reafirma la necesidad de contar con un instrumento que facilite su medición.

Por su parte, Sánchez (2018) plantea que el concepto de satisfacción "parece estar asociada directa y cercanamente a las nociones de cumplimiento de expectativas, necesidades y deseos, bienestar, confort, felicidad, placidez, placer, encantamiento, contentamiento, alegría, equilibrio y deleite" (p. 22); por lo tanto, resulta interesante conocer cuáles son las variables que lo pueden integrar, y a su vez, entender la manera en la que este elemento puede vincularse con otros muy importantes para el estudiantado, como lo es el desempeño académico.

En vista de la importancia que tiene el estudio de esta variable, los investigadores Gento y Vivas, en el 2003 desarrollaron un instrumento para medir la satisfacción de los estudiantes universitarios con su educación, llamado SEUE, el cual lo constituyen 93 preguntas agrupadas en diez dimensiones y fue validado solo por medio del uso del Alfa de Cronbach. Dicho instrumento se diseñó y aplicó en la Universidad del Táchira, la cual es una institución autónoma venezolana de carácter público; por lo 
tanto, se decidió aplicar el constructo para su validación a estudiantes de la Universidad del Zulia, ya que es una Universidad del mismo tipo, con características estructurales y funcionamiento similares.

A continuación, en la tabla 1, se presenta una breve descripción de las dimensiones que lo integran. Como se puede observar, el instrumento cuenta con algunas dimensiones similares, por lo que se hace interesante conocer la posibilidad de reducirlas, con el fin de facilitar los análisis posteriores

TABLA 1

Definición de las dimensiones

\begin{tabular}{|c|c|}
\hline Dimensión & Descripción \\
\hline $\begin{array}{l}\text { 1. Condiciones básicas } \\
\text { de la infraestructura }\end{array}$ & $\begin{array}{l}\text { Mide la satisfacción de los estudiantes con respecto a las características físicas } \\
\text { de las instalaciones, desde las condiciones de la infraestructura, hasta la } \\
\text { existencia, equipamiento y adecuación de cada área para las actividades que } \\
\text { en ellas se desarrollan. }\end{array}$ \\
\hline 2. Servicios de la institución. & $\begin{array}{l}\text { Trata acerca de la calidad y cantidad de servicios estudiantiles y de apoyo que } \\
\text { ofrece la institución para ayudar al estudiantado. }\end{array}$ \\
\hline $\begin{array}{l}\text { 3. Condiciones } \\
\text { de seguridad }\end{array}$ & $\begin{array}{l}\text { Indaga sobre la satisfacción de los alumnos por tener la seguridad de que } \\
\text { las condiciones de supervivencia básica, dentro del entorno habitual de la } \\
\text { institución están garantizadas. }\end{array}$ \\
\hline $\begin{array}{l}\text { 4. Consideración a su } \\
\text { situación económica }\end{array}$ & $\begin{array}{l}\text { Mide la satisfacción del alumnado por sentir que su condición socioeconómica } \\
\text { es tomada en consideración y no constituye un obstáculo para su proceso } \\
\text { formativo. }\end{array}$ \\
\hline $\begin{array}{l}\text { 5. Seguridad emocional } \\
\text { por el trato afectuoso }\end{array}$ & $\begin{array}{l}\text { Evalúa la satisfacción por contar con el trato afectuoso que requiere el desarrollo } \\
\text { equilibrado de la personalidad, e involucra tanto al profesorado, como a sus } \\
\text { compañeros, autoridades, personal administrativo y de servicios. }\end{array}$ \\
\hline 6. Sentido de pertenencia & $\begin{array}{l}\text { Trata sobre la satisfacción por contar con el reconocimiento y la aceptación por } \\
\text { el hecho de ser miembros de la comunidad estudiantil y del grupo, así como } \\
\text { tener las oportunidades de participar en las políticas y proyectos institucionales. }\end{array}$ \\
\hline $\begin{array}{l}\text { 7. Proceso de enseñanza } \\
\text { aprendizaje }\end{array}$ & $\begin{array}{l}\text { Medida a través de la satisfacción que perciben los estudiantes de los factores } \\
\text { que intervienen en la adquisición de sus conocimientos e incluye la metodología } \\
\text { y técnicas de evaluación empleados. }\end{array}$ \\
\hline 8. Logros personales & $\begin{array}{l}\text { Mide la satisfacción de los estudiantes con respecto a las calificaciones } \\
\text { obtenidas, y su desarrollo personal y profesional. }\end{array}$ \\
\hline $\begin{array}{l}\text { 9. Reconocimiento } \\
\text { al éxito personal }\end{array}$ & $\begin{array}{l}\text { Se refiere a la satisfacción por el reconocimiento por parte de las autoridades y } \\
\text { superiores sobre los logros obtenidos por el estudiantado. }\end{array}$ \\
\hline 10. Autorrealización & $\begin{array}{l}\text { Mide la satisfacción con respecto a los conocimientos, habilidades y valores } \\
\text { adquiridos y por la capacitación recibida para su futura inserción al mundo } \\
\text { laboral. }\end{array}$ \\
\hline
\end{tabular}

Fuente: Adaptado de Gento et al. (2003). 
El propósito principal de la presente investigación es validar cualitativa y cuantitativamente el instrumento desarrollado por Gento y Vivas (2003), para así probar en forma adicional su reproducibilidad y estabilidad, en aras de obtener un constructo que pueda ser aplicado a cualquier población estudiantil y en cualquier momento del tiempo, para complementar el estudio de la calidad educativa y poder relacionar los resultados obtenidos con otras variables importantes, tales como: el desempeño académico y las condiciones de confort presentes en las instituciones de educación superior.

\section{MATERIALES Y MÉTODOS}

Para el desarrollo de la presente investigación, se inició empleando el juicio de expertos para lograr la validez cualitativa del mismo, posteriormente se calculó el tamaño de la muestra para una población finita o de tamaño conocido conformada por 2.653 estudiantes activos de la Facultad de Ingeniería de la Universidad del Zulia, para un error del $5 \%$ y confiabilidad del $95 \%$, la cual resultó en 336 estudiantes, a quienes se les aplicó aleatoriamente con la finalidad de realizar las pruebas pertinentes.

Para iniciar, se calculó la confiabilidad del instrumento empleando el Alfa de Cronbach, el cual refleja la consistencia interna del constructo; es decir, permite conocer la manera como todos los ítems covarían entre sí. Al respecto, Celina et al. (2005) plantean que el valor mínimo aceptable para el coeficiente Alfa de Cronbach es 0.7. Luego, resultó necesario emplear la prueba de correlación de Pearson para conocer la interacción de cada uno de los indicadores con el total, y así entender el aporte que cada uno de los ítems medidos ofrece al análisis de la satisfacción de los estudiantes con el empleo del presente instrumento. En forma adicional, se realizó un análisis factorial exploratorio para determinar la validez del constructo y lograr reducir las dimensiones diseñadas al inicio y así fusionar a aquellas con propósitos similares.

Sin embargo, el uso de los análisis anteriores permite estudiar solo la consistencia interna del constructo, por lo que para conocer la posibilidad de emplearlo en otros momentos del tiempo y poblaciones diferentes (estabilidad y reproducibidad), se requieren otros tipos de análisis. En función de esto, se procedió a determinar inicialmente la validez de criterio (validez concurrente), la cual consiste en comprobar si el instrumento evaluado mide en realidad la satisfacción estudiantil; para lograrlo, se compararon los resultados obtenidos al aplicarlo con los alcanzados al usar un instrumento patrón a una parte de la misma muestra, en este caso se encontró uno diseñado y aplicado en la Universidad de Tolima para determinar el grado de satisfacción de los estudiantes con la institución. Luego, se compararon los niveles de satisfacción obtenidos en cada instrumento para confirmar que la percepción de la muestra fuera consistente.

Posteriormente se consideró necesario determinar la estabilidad del instrumento, para lo cual se aplicó el mismo constructo a una parte de la muestra dos veces, con dos meses de diferencia entre ambas; luego se empleó el coeficiente R de Pearson y el análisis de la varianza para comprobar si los resultados eran consistentes; pues estos índices permiten conocer el grado de correlación entre dos variables. En este caso, son las percepciones de satisfacción del estudiantado en dos momentos del tiempo.

Finalmente, se determinó el rendimiento del constructo a través del uso de la curva COR. Para lograrlo, se dicotomizaron los resultados obtenidos al aplicar el cuestionario entre estudiantes satisfechos e insatisfechos. La ventaja del uso de esta prueba, es que permite determinar la razón de verdaderos positivos y verdaderos negativos, con lo cual se evidencia la sensibilidad y la especificidad del instrumento evaluado. Con estos resultados, se empleó el Índice de Youden para calcular el punto de corte óptimo; es decir, conocer el puntaje que debía obtener el alumnado para considerarse satisfechos o instatisfechos con las condiciones de la institución. 


\section{DISCUSIÓN DE RESULTADOS}

Inicialmente, se realizó un análisis al instrumento SEUE desarrollado por Gento Palacios y Vivas García en el 2003. Este cuestionario consta de 93 preguntas, con una escala de valoración del 1 al 5, donde 1 significa muy insatisfecho y el 5 muy satisfecho, divididas en diez dimensiones. Para su revisión, se empleó la técnica de Juicio de Expertos para lograr la validación cualitativa; sin embargo, luego del análisis, se determinó que el constructo en su primera dimensión, relativa a las Condiciones Básicas de la Infraestructura, no poseía preguntas relativas a la lluminación de las áreas de estudio, a pesar de poseer indicadores para el resto de las variables ambientales comúnmente estudiadas; por lo tanto, se procedió a incorporar un ítem relativo a este, de tal forma se obtuvo un instrumento definitivo cuyas características se muestran en la Tabla 2.

TABLA 2

Resumen de los datos del instrumento

\begin{tabular}{|c|c|c|}
\hline \multirow{3}{*}{$\begin{array}{c}\text { Valores } \\
\text { (Escala de Lickert) }\end{array}$} & $1=$ Muy insatisfecho & $4=$ Satisfecho \\
\hline & $2=$ Insatisfecho & \multirow{2}{*}{$5=$ Muy satisfecho } \\
\hline & $3=$ Poco satisfecho & \\
\hline \multirow{5}{*}{ Dimensiones } & $\begin{array}{l}\text { 1. Condiciones básicas de la } \\
\text { infraestructura }\end{array}$ & 6. Sentido de pertenencia \\
\hline & 2. Servicios de la institución. & $\begin{array}{l}\text { 7. Proceso de enseñanza } \\
\text { aprendizaje }\end{array}$ \\
\hline & 3. Condiciones de seguridad & 8. Logros personales \\
\hline & $\begin{array}{l}\text { 4. Consideración a su } \\
\text { situación económica }\end{array}$ & $\begin{array}{l}\text { 9. Reconocimiento al éxito } \\
\text { personal }\end{array}$ \\
\hline & $\begin{array}{l}\text { 5. Seguridad emocional por } \\
\text { el trato afectuoso }\end{array}$ & 10. Autorrealización \\
\hline Cantidad de ítems & \multicolumn{2}{|l|}{94 ítems } \\
\hline Muestra & \multicolumn{2}{|l|}{336 estudiantes } \\
\hline
\end{tabular}

Luego, el instrumento fue aplicado aleatoriamente a una muestra tomada de los 2.653 estudiantes activos de la Facultad de Ingeniería de la Universidad del Zulia, para un error del $5 \%$ y confiabilidad del 95 \%, la cual resultó en 336 estudiantes. Debido a que se incluyó un ítem relativo a las condiciones de lluminación, y se aplicó el cuestionario en otra entidad educativa, se procedió a calcular el Alfa de Cronbach, cuyo coeficiente resultó 0,964 . 
De acuerdo con Celina et al. (2005), la confiabilidad del instrumento resultó muy alta; por lo tanto, se determinó que la incorporación de la pregunta adicional no afectó negativamente a la fiabilidad de este y se decidió que sería el definitivo para la realización del presente estudio. Luego de obtener la confiabilidad del instrumento, se procedió a efectuar la prueba de correlación de Pearson, para determinar el aporte de cada ítem con respecto a la satisfacción total, la Tabla 3 presenta el resultado:

TABLA 3

Correlaciones instrumento con satisfacción total

\begin{tabular}{|c|c|c|c|c|c|c|c|c|c|c|c|c|c|c|c|c|c|c|c|c|}
\hline & ITEM & \begin{tabular}{|l|} 
SUM \\
TOTAL
\end{tabular} & ITEM & $\begin{array}{l}\text { SUM } \\
\text { TOTAL }\end{array}$ & ITEM & $\begin{array}{l}\text { SUM } \\
\text { TOTAL }\end{array}$ & ITEM & $\begin{array}{l}\text { SUM } \\
\text { TOTAL }\end{array}$ & ITEM & $\begin{array}{l}\text { SUM } \\
\text { TOTAL }\end{array}$ & ITEM & $\begin{array}{l}\text { SUM } \\
\text { TOTAL }\end{array}$ & ITEM & $\begin{array}{l}\text { SUM } \\
\text { TOTAL }\end{array}$ & ITEM & \begin{tabular}{|l|} 
SUM \\
TOTAL
\end{tabular} & ITEM & $\begin{array}{l}\text { SUM } \\
\text { TOTAL }\end{array}$ & ITEM & \begin{tabular}{|l|} 
SUM \\
TOTAL
\end{tabular} \\
\hline $\begin{array}{l}\text { Cor. Pearson } \\
\text { Sig. (bilateral) }\end{array}$ & 101 & \begin{tabular}{r|}
, 316 \\
, 000 \\
336
\end{tabular} & 111 & \begin{tabular}{r|}
.460 \\
.000 \\
336 \\
\end{tabular} & 121 & \begin{tabular}{r|}
, 444 \\
, 000 \\
336 \\
\end{tabular} & 131 & \begin{tabular}{r|}
, 486 \\
, 000 \\
336
\end{tabular} & 141 & \begin{tabular}{l|}
, 513 \\
, 000 \\
336
\end{tabular} & 151 & $\begin{array}{l}, 464 \\
.000 \\
336\end{array}$ & 161 & $\begin{array}{l}, 266 \\
, 000 \\
336\end{array}$ & 171 & \begin{tabular}{r|}
, 536 \\
.000 \\
336
\end{tabular} & 181 & \begin{tabular}{l|}
, 359 \\
, 000 \\
336 \\
\end{tabular} & 191 & \begin{tabular}{l|}
, 504 \\
, 000 \\
336
\end{tabular} \\
\hline $\begin{array}{l}\text { Cor. Pearson } \\
\text { Sig. (bilateral) } \\
N\end{array}$ & 102 & \begin{tabular}{r|}
, 395 \\
, 000 \\
336
\end{tabular} & 112 & $\begin{array}{r}, 386 \\
, 000 \\
336\end{array}$ & 122 & $\begin{array}{l}, 445 \\
.000 \\
336\end{array}$ & 132 & $\begin{array}{l}.446 \\
.000 \\
336\end{array}$ & 142 & \begin{tabular}{r|}
, 244 \\
.000 \\
336
\end{tabular} & 152 & \begin{tabular}{r|}
, 516 \\
.000 \\
336
\end{tabular} & 162 & \begin{tabular}{r|}
, 455 \\
.000 \\
336
\end{tabular} & 172 & $\begin{array}{r}.474 \\
.000 \\
336\end{array}$ & 182 & \begin{tabular}{r|}
, 436 \\
.000 \\
336
\end{tabular} & 192 & $\begin{array}{l}, 482 \\
.000 \\
336\end{array}$ \\
\hline $\begin{array}{l}\text { Cor. Pearson } \\
\text { Sig. (bilateral) } \\
N\end{array}$ & 103 & \begin{tabular}{r|}
, 416 \\
.000 \\
336
\end{tabular} & 113 & $\begin{array}{r}.458 \\
, 000 \\
336\end{array}$ & 123 & $\begin{array}{l}.453 \\
.000 \\
336\end{array}$ & 133 & $\begin{array}{r}.425 \\
, 000 \\
336\end{array}$ & 143 & \begin{tabular}{|l|}
.422 \\
.000 \\
336
\end{tabular} & 153 & $\begin{array}{r}, 530 \\
, 000 \\
336\end{array}$ & 163 & $\begin{array}{r}, 494 \\
, 000 \\
336\end{array}$ & 173 & \begin{tabular}{r|}
, 357 \\
, 000 \\
336
\end{tabular} & 183 & \begin{tabular}{r|}
, 373 \\
.000 \\
336
\end{tabular} & 193 & $\begin{array}{l}, 431 \\
.000 \\
336\end{array}$ \\
\hline $\begin{array}{l}\text { Cor. Pearson } \\
\text { Sig. (bilateral) } \\
N\end{array}$ & 104 & $\begin{array}{r}, 379 \\
.000 \\
336 \\
\end{array}$ & 114 & $\begin{array}{r}401 \\
.000 \\
336 \\
\end{array}$ & 124 & $\begin{array}{l}471 \\
.000 \\
336 \\
\end{array}$ & 134 & $\begin{array}{r}, 550 \\
, 000 \\
336\end{array}$ & 144 & \begin{tabular}{r|}
, 511 \\
.000 \\
336
\end{tabular} & 154 & $\begin{array}{r}, 406 \\
.000 \\
336\end{array}$ & 164 & $\begin{array}{r}, 419 \\
.000 \\
336\end{array}$ & 174 & \begin{tabular}{r|}
, 520 \\
, 000 \\
336
\end{tabular} & 184 & \begin{tabular}{r|}
, 541 \\
.000 \\
336
\end{tabular} & 194 & $\begin{array}{l}, 337 \\
, 000 \\
336\end{array}$ \\
\hline $\begin{array}{l}\text { Cor. Pearson } \\
\text { Sig. (bilateral) } \\
N\end{array}$ & 105 & \begin{tabular}{r|}
, 535 \\
.000 \\
336
\end{tabular} & 115 & $\begin{array}{r}.421 \\
.000 \\
336\end{array}$ & 125 & $\begin{array}{l}.480 \\
.000 \\
336\end{array}$ & 135 & $\begin{array}{r}, 542 \\
, 000 \\
336\end{array}$ & 145 & \begin{tabular}{r|}
547 \\
.000 \\
336
\end{tabular} & 155 & $\begin{array}{r}, 450 \\
, 000 \\
336\end{array}$ & 165 & $\begin{array}{r}.401 \\
.000 \\
336\end{array}$ & 175 & \begin{tabular}{r|}
, 546 \\
.000 \\
336
\end{tabular} & 185 & \begin{tabular}{r|}
, 474 \\
.000 \\
336
\end{tabular} & & \\
\hline $\begin{array}{l}\text { Cor. Pearson } \\
\text { Sig. (bilateral) } \\
N\end{array}$ & 106 & \begin{tabular}{r|}
, 549 \\
.000 \\
336
\end{tabular} & 116 & $\begin{array}{r}.438 \\
.000 \\
336\end{array}$ & 126 & $\begin{array}{l}.442 \\
.000 \\
336\end{array}$ & 136 & $\begin{array}{r}.486 \\
.000 \\
336\end{array}$ & 146 & \begin{tabular}{r|}
.499 \\
.000 \\
336
\end{tabular} & 156 & $\begin{array}{r}.518 \\
.000 \\
336\end{array}$ & 166 & $\begin{array}{r}.450 \\
.000 \\
336\end{array}$ & 176 & \begin{tabular}{r|}
, 518 \\
, 000 \\
336
\end{tabular} & 186 & \begin{tabular}{r|}
, 442 \\
.000 \\
336
\end{tabular} & & \\
\hline $\begin{array}{l}\text { Cor. Pearson } \\
\text { Sig. (bilateral) } \\
N\end{array}$ & 107 & $\begin{array}{r}, 396 \\
.000 \\
336\end{array}$ & 117 & $\begin{array}{r}, 523 \\
.000 \\
336\end{array}$ & 127 & \begin{tabular}{|l|}
, 460 \\
.000 \\
336
\end{tabular} & 137 & $\begin{array}{r}, 493 \\
.000 \\
336\end{array}$ & 147 & \begin{tabular}{r|}
.535 \\
.000 \\
336
\end{tabular} & 157 & $\begin{array}{r}.537 \\
.000 \\
336\end{array}$ & 167 & \begin{tabular}{r|}
, 490 \\
, 000 \\
336
\end{tabular} & 177 & \begin{tabular}{r|}
, 475 \\
.000 \\
336
\end{tabular} & 187 & \begin{tabular}{r|}
, 437 \\
.000 \\
336
\end{tabular} & & \\
\hline $\begin{array}{l}\text { Cor. Pearson } \\
\text { Sig. (bilateral) } \\
N\end{array}$ & 108 & \begin{tabular}{r|}
, 209 \\
.000 \\
336
\end{tabular} & 118 & $\begin{array}{r}., 396 \\
.000 \\
336\end{array}$ & 128 & $\begin{array}{l}.420 \\
.000 \\
336\end{array}$ & 138 & $\begin{array}{r}., 570 \\
.000 \\
336\end{array}$ & 148 & \begin{tabular}{r|}
.450 \\
.000 \\
336
\end{tabular} & 158 & \begin{tabular}{r|}
, 449 \\
.000 \\
336
\end{tabular} & 168 & $\begin{array}{r}, 532 \\
, 000 \\
336\end{array}$ & 178 & \begin{tabular}{r|}
, 301 \\
.000 \\
336
\end{tabular} & 188 & $\begin{array}{l}, 497 \\
.000 \\
336\end{array}$ & & \\
\hline $\begin{array}{l}\text { Cor. Pearson } \\
\text { Sig. (bilateral) } \\
N\end{array}$ & 109 & $\begin{array}{r}.304 \\
.000 \\
336\end{array}$ & 119 & $\begin{array}{r}.409 \\
.000 \\
336\end{array}$ & 129 & $\begin{array}{r}.483 \\
.000 \\
336\end{array}$ & 139 & $\begin{array}{r}.417 \\
.000 \\
336\end{array}$ & 149 & \begin{tabular}{r|}
.536 \\
.000 \\
335
\end{tabular} & 159 & $\begin{array}{r}.434 \\
.000 \\
336\end{array}$ & 169 & $\begin{array}{r}.457 \\
.000 \\
336\end{array}$ & 179 & \begin{tabular}{r|}
, 295 \\
.000 \\
336
\end{tabular} & 189 & \begin{tabular}{r|}
483 \\
.000 \\
336
\end{tabular} & & \\
\hline $\begin{array}{l}\text { Cor. Pearson } \\
\text { Sig. (bilateral) } \\
N\end{array}$ & 110 & \begin{tabular}{r|}
, 513 \\
.000 \\
336
\end{tabular} & 120 & $\begin{array}{r}.352 \\
.000 \\
336\end{array}$ & 130 & $\begin{array}{l}.446 \\
.000 \\
336\end{array}$ & 140 & $\begin{array}{r}, 526 \\
.000 \\
336\end{array}$ & 150 & \begin{tabular}{|l|}
.542 \\
.000 \\
336
\end{tabular} & 160 & $\begin{array}{r}.434 \\
.000 \\
336\end{array}$ & 170 & $\begin{array}{r}, 531 \\
.000 \\
336\end{array}$ & 180 & \begin{tabular}{r|}
, 340 \\
.000 \\
336
\end{tabular} & 190 & \begin{tabular}{r|}
.413 \\
.000 \\
336
\end{tabular} & & \\
\hline
\end{tabular}

Fuente: Elaboración propia.

Como se puede observar, todos los ítems tienen una alta correlación, la cual oscila entre 0,209 y 0,570, y una significancia menor a 0,05, por lo que se acepta el uso de todos los indicadores; pues todos contribuyen en forma positiva a la medición de la satisfacción estudiantil

\section{Análisis factorial exploratorio (validez del constructo)}

Para iniciar el Análisis Factorial, se procedió a revisar ítem por ítem, esto consistió en un análisis de las correlaciones existentes entre cada uno de los indicadores evaluados con respecto a los 93 restantes. Luego del análisis correspondiente, se determinó que existían 827 relaciones significativas (o superiores al 0,300) de los 4171 cruces efectuados, lo cual representa el 18,92\%. 
Con estos datos, se procedió a calcular el valor del determinante de la matriz de correlaciones y se realizó la prueba de esfericidad de Barlett y la medida de adecuación de la muestra de Kaiser-Meyer-Olkin (KMO), para conocer si las variables en estudio se encontraban suficientemente correlacionadas entre sí, de ello resultó la tabla 4 mostrada a continuación:

TABLA 4

KMO y prueba de Bartlett

\begin{tabular}{|l|c|r|}
\hline Medida de adecuación muestral de Kaiser-Meyer-Olkin. & ,909 \\
\hline Prueba de esfericidad de Bartlett & Chi-cuadrado aproximado & $\mathbf{2 2 4 7 0 , 8 8 9}$ \\
& Gl & $\mathbf{4 3 7 1}$ \\
\hline & Sig. &, 000 \\
\hline
\end{tabular}

Fuente: Elaboración propia.

El valor del KMO resultó 0,909, el cual se considera muy alto según la escala del Baremo de Kaiser; por lo tanto, se puede concluir que existe correlación entre los ítems estudiados y se puede proceder a efectuar el análisis factorial. Por su parte, la prueba de Barlett arroja un nivel de significancia igual a 0,000, por lo cual se rechaza la hipótesis nula que planteaba igualdad entre los ítems y se puede inferir que por el contrario, los ítems si están interrelacionados.

Luego de haber comprobado la interrelación entre los factores, se procedió a efectuar la extracción de estos, para reducir la cantidad de dimensiones, al haber encontrado que algunas de ellas se relacionaban entre sí. Para lograrlo, se calculó la matriz de componentes rotados para la extracción de factores y se indicó que se deseaba reducir a seis la cantidad de dimensiones del constructo. Se encontraron catorce ítems con problemas de ubicación, los cuales serán analizados posteriormente para observar en cuál dimensión encajan mejor, de acuerdo con su definición teórica y funcional, se presentan en la Tabla 5:

TABLA 5

Ítems con conflicto de Ubicación

\begin{tabular}{|c|c|}
\hline $\begin{array}{c}\text { Ítems con doble carga } \\
16,136,141,151,170\end{array}$ & $\begin{array}{l}\text { Ítems con baja correlación (menor a 0,4) } \\
11,18,19,118,119,120,121,125,142,143,161,162,184,187\end{array}$ \\
\hline
\end{tabular}

En función de los resultados obtenidos del análisis anterior, las dimensiones resultantes del constructo aplicado quedaron distribuidas como se muestra en la Tabla 6.

TABLA 6

Explicación de Factores

\begin{tabular}{|c|c|c|}
\hline Dimensión & Nombre & Ítems que la integran \\
\hline Dimensión 1 & Condiciones físicas & $12, I 3,14,15,110,111, \mid 14$, I27, I28, I29, I30, I31, I32, I33 \\
\hline Dimensión 2 & Servicios estudiantiles & $17,|12,113,115,116| 117,122,123,124,146,147$. \\
\hline Dimensión 3 & Consideración a limitaciones económicas & 126, I34, 135, I37, 138, 139, 140, 148 \\
\hline Dimensión 4 & Relaciones personales & $144,145,149,150,152,153,154,155,156,157,158$. \\
\hline Dimensión 5 & Proceso enseñanza - aprendizaje & $\begin{array}{c}159,160,164,165,166,167,168,169,173,178,179,180,181,182, \\
183,185,186,188,189,190,191,192,193,194 .\end{array}$ \\
\hline Dimensión 6 & Inserción al contexto mundial & $163,171,172,174,175,176,177$ \\
\hline
\end{tabular}

Fuente: Elaboración propia. 
La primera dimensión, llamada Condiciones Físicas, es uno de los factores más investigados, y entre sus elementos se hallan las condiciones ambientales, así como diferentes aspectos del ambiente físico. De acuerdo con un amplio estudio desarrollado por Higgins y colaboradores (2005), los elementos físicos del medio ambiente de estudio han mostrado tener efectos discernibles tanto en profesores como en alumnos. En particular, un control inadecuado de muchos de estos factores tienen efectos adversos en la concentración, humor, sensación de bienestar, asistencia y logros (p.6).

Los Servicios Estudiantiles se refieren a todos los procesos que se desarrollan dentro de la Universidad fuera del aspecto académico, como Servicio de Comedores, Oficinas de Atención a los Estudiantes, entre otros. Por su parte Gento y Vivas (2003) definen esta dimensión como Satisfacción de los alumnos con la manera en que funcionan los servicios estudiantiles (p.6).

En cuanto a la dimensión Consideración a limitaciones económicas, se refiere a la posibilidad de los estudiantes de acceder a los servicios de la institución y poder cumplir con las exigencias hechas, muchas veces en clase, las cuales generan un impacto económico en los discentes. Es un aspecto relevante, en especial al efectuarse el estudio en una institución de carácter público, y al respecto, Albarran-Peña (2019) encontró que el 50 \% de los estudiantes han desertado de la Universidad de Los Andes y expone que entre las causas se encuentran la falta de oportunidades laborales, aumento de los gastos personales y académicos, carencia de recursos económicos, entre otros (p.3).

La dimensión Relaciones Personales, involucra no solo al trato con sus compañeros, sino también con profesores, personal administrativo y todos aquellos quienes forman parte del entorno universitario. En cuanto a este elemento, Medina et al. (2018) concluyeron que algunos factores personales, como los relacionados con duelos, relaciones de pareja, entre otros, pueden afectar el rendimiento académico de los alumnos (p.23); por lo tanto, constituye un aspecto de importancia que debe investigarse.

El proceso de Enseñanza-Aprendizaje, se refiere a la manera en la cual se imparten las ;actividades académicas, por lo tanto, se puede indicar que constituye el aspecto medular de las instituciones educativas. Al respecto, en un caso de estudio desarrollado por Basantes (2017) en la Universidad Nacional Chimborazo Riobamba, en Ecuador, el investigador concluyó que existía una alta influencia entre la calidad de los servicios educativos y la satisfacción general de los estudiantes (p.92), se constituye en un punto vital a ser analizado al estudiar la satisfacción estudiantil.

Finalmente, la dimensión Inserción al contexto mundial, se refiere no solo a la enseñanza de técnicas modernas que se usan en el mercado laboral, que cada vez es más competitivo, sino también al empleo de herramientas actualizadas que faciliten los procesos académicos. Con respecto a esto, Potthoff (2009) planteó que la mayor dificultad con la que se deben enfrentar los usuarios, se encuentra en que el personal o los estudiantes no reciben el entrenamiento necesario para su uso (p.6) y se les dificulta su manejo, lo cual conlleva a otro factor que debe ser estudiado, el soporte técnico, este se constituye en un elemento muy importante en las escuelas para poder mantener la tecnología de información; pues requiere estabilidad. 
En virtud a la definición dada a cada una de las dimensiones, se procedió a disponer los ítems con problemas de ubicación, el instrumento final quedó organizado de la siguiente manera (Tabla 7):

TABLA 7

Distribución de Factores definitiva

\begin{tabular}{|c|c|c|}
\hline Dimensión & Item & Planteamiento \\
\hline Dimensión 1 & $\begin{array}{l}101 \\
102 \\
103 \\
104 \\
105 \\
106 \\
108 \\
109 \\
110 \\
111 \\
114 \\
127 \\
128 \\
129 \\
130 \\
131 \\
132 \\
133\end{array}$ & $\begin{array}{l}\text { Limpieza de las instalaciones } \\
\text { Ventilación de las aulas } \\
\text { Aislamiento de los ruidos } \\
\text { Iluminación de las áreas de estudio } \\
\text { Comodidad del mobiliario } \\
\text { Espacios para la enseñanza } \\
\text { Instalaciones sanitarias } \\
\text { Disponibilidad de agua potable } \\
\text { Espacios para el descanso } \\
\text { Espacios para la recreación } \\
\text { Instalaciones deportivas } \\
\text { Transporte } \\
\text { Del edificio (salidas de emergencia, existencia de extintores) } \\
\text { Del mobiliario (sin aristas peligrosas, materiales rompibles o inflamables) } \\
\text { Del transporte (del vehículo, de la ruta) } \\
\text { De las zonas de esparcimiento } \\
\text { De las instalaciones deportivas } \\
\text { De los alrededores de la institución }\end{array}$ \\
\hline Dimensión & Item & Planteamiento \\
\hline Dimensión 2 & $\begin{array}{l}107 \\
112 \\
113 \\
115 \\
116 \\
117 \\
118 \\
121 \\
122 \\
123 \\
124 \\
125 \\
146 \\
147 \\
162\end{array}$ & $\begin{array}{l}\text { Espacios para el estudio } \\
\text { Instalaciones de la biblioteca } \\
\text { Instalaciones del comedor } \\
\text { Servicio bibliotecario (atención al usuario) } \\
\text { Dotación de la biblioteca (cantidad y vigencia de los libros y revistas) } \\
\text { Oficina de registro y control estudiantil } \\
\text { Proceso de admisión e inscripción } \\
\text { Servicio de reproducción y fotocopiado } \\
\text { Higiene de los alimentos en el comedor } \\
\text { Cantidad de alimentos ofrecidos } \\
\text { Calidad de la alimentación } \\
\text { El apoyo que brinda el centro de estudiantes } \\
\text { El personal de la biblioteca } \\
\text { El personal del comedor } \\
\text { Proceso de admisión e inscripción }\end{array}$ \\
\hline Dimensión & Item & Planteamiento \\
\hline Dimensión 3 & $\begin{array}{l}126 \\
134\end{array}$ & $\begin{array}{l}\text { Servicios médico-odontológicos } \\
\text { Las actividades académicas }\end{array}$ \\
\hline
\end{tabular}




\begin{tabular}{|c|c|c|}
\hline Dimensión & Item & Planteamiento \\
\hline Dimensión 3 & $\begin{array}{l}135 \\
136 \\
137 \\
138 \\
139 \\
140 \\
148\end{array}$ & $\begin{array}{l}\text { Las actividades extra cátedras } \\
\text { El servicio de comedor } \\
\text { El servicio de transporte } \\
\text { Los servicios médico-asistenciales } \\
\text { La asignación de becas } \\
\text { La adquisición de materiales de estudio } \\
\text { El personal del servicio médico }\end{array}$ \\
\hline Dimensión & Item & Planteamiento \\
\hline Dimensión 4 & $\begin{array}{l}142 \\
143 \\
144 \\
145 \\
149 \\
150 \\
151 \\
152 \\
153 \\
154 \\
155 \\
156 \\
157 \\
158 \\
184\end{array}$ & $\begin{array}{l}\text { Sus compañeros de grupo } \\
\text { Los otros alumnos de la universidad } \\
\text { Las autoridades de la universidad } \\
\text { El personal administrativo } \\
\text { Las autoridades } \\
\text { Los jefes de departamentos } \\
\text { Trato recibido de sus profesores } \\
\text { El personal administrativo } \\
\text { El personal de servicios } \\
\text { Los compañeros de clase } \\
\text { El contexto social por ser estudiante de la institución } \\
\text { Consideración y respeto a sus ideas y propuestas en el aula } \\
\text { Representación en las diversas instancias de la institución } \\
\text { Participación en las políticas y proyectos institucionales } \\
\text { La institución y sus autoridades }\end{array}$ \\
\hline Dimensión & Item & Planteamiento \\
\hline Dimensión 5 & $\begin{array}{l}141 \\
159 \\
160 \\
161 \\
164 \\
165 \\
166 \\
167 \\
168 \\
169 \\
173 \\
178 \\
179 \\
180 \\
181 \\
182 \\
183\end{array}$ & $\begin{array}{l}\text { Sus profesores } \\
\text { El plan de estudios de la carrera } \\
\text { El contenido de la asignatura } \\
\text { El régimen de estudios (anual/semestral) } \\
\text { Dominio de los contenidos y actualización de los profesores } \\
\text { El nivel de exigencia } \\
\text { El sistema de evaluación } \\
\text { La orientación y apoyo de los profesores en las horas de consulta } \\
\text { La metodología de enseñanza } \\
\text { La comunicación con los profesores en el aula } \\
\text { La asistencia a clase de los profesores } \\
\text { Con los resultados o calificaciones obtenidas } \\
\text { Con los conocimientos adquiridos } \\
\text { Con el dominio de técnicas de estudio y trabajo intelectual alcanzadas } \\
\text { Con la formación ciudadana obtenida (valores desarrollados) } \\
\text { Con las habilidades y destrezas para el trabajo docente desarrolladas } \\
\text { Con el desarrollo integral de su persona }\end{array}$ \\
\hline
\end{tabular}




\begin{tabular}{|c|c|c|}
\hline Dimensión & Item & Planteamiento \\
\hline Dimensión 5 & $\begin{array}{l}185 \\
186 \\
187 \\
188 \\
189 \\
190 \\
191 \\
192 \\
193 \\
194\end{array}$ & $\begin{array}{l}\text { Los profesores } \\
\text { Los alumnos de su grupo } \\
\text { Los demás alumnos de la universidad } \\
\text { El contexto social } \\
\text { Poder hacer lo que verdaderamente quiere y está en capacidad de hacer } \\
\text { Saber que cada día va adquiriendo mayores conocimientos y destrezas } \\
\text { La libertad que goza en la institución } \\
\text { La autonomía de la que goza en la realización de sus trabajos } \\
\text { El desarrollo de su creatividad } \\
\text { Su futuro profesional }\end{array}$ \\
\hline Dimensión & Item & Planteamiento \\
\hline Dimensión 6 & $\begin{array}{l}119 \\
120 \\
163 \\
170 \\
171 \\
172 \\
174 \\
175 \\
176 \\
177\end{array}$ & $\begin{array}{l}\text { Acceso a computadoras para realizar trabajos académicos } \\
\text { Acceso a internet y correo electrónico } \\
\text { Los materiales impresos y audiovisuales disponibles } \\
\text { La formación práctica } \\
\text { La vinculación con los futuros centros de trabajo } \\
\text { La vinculación con el contexto nacional y regional } \\
\text { El acceso a las diversas manifestaciones culturales (teatro, cine, } \\
\text { conciertos) } \\
\text { Los cursos extra cátedras para optimizar su formación } \\
\text { Incorporación de las nuevas tecnologías a la enseñanza } \\
\text { El servicio de asistencia y asesoramiento psicológico }\end{array}$ \\
\hline
\end{tabular}

Fuente: Elaboración propia.

Luego de haber ubicado todos los ítems, se procedió a determinar la confiabilidad de cada una de las dimensiones, por medio del cálculo del Alfa de Cronbach a cada una de ellas para confirmar la correlación entre los indicadores que las conforman. Los resultados obtenidos se presentan en la Tabla 8:

TABLA 8

Resumen de resultados análisis factorial

\begin{tabular}{|c|c|c|c|c|}
\hline Dimensión & Nombre & Ítems que la integran & $\begin{array}{c}\# \\
\text { Ítems }\end{array}$ & $\begin{array}{l}\text { Alfa de } \\
\text { Cronbach }\end{array}$ \\
\hline Dimensión 1 & Condiciones físicas & $\begin{array}{c}11,12,13,14,15,16,18,19,110,111,114,127 \\
128,129,130,131,132,133\end{array}$ & 18 & 0.879 \\
\hline Dimensión 2 & Servicios estudiantiles & 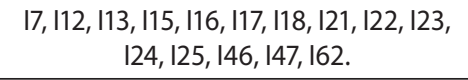 & 15 & 0.871 \\
\hline Dimensión 3 & $\begin{array}{l}\text { Accesibilidad económica } \\
\text { a servicios }\end{array}$ & I26, I34, I35, I36, I37, I38, I39, I40, 148. & 9 & 0.862 \\
\hline Dimensión 4 & Relaciones personales & $\begin{array}{c}142,143,144,145,149,150,151,152,153, \\
154,155,156,157,158,184 .\end{array}$ & 15 & 0.882 \\
\hline Dimensión 5 & $\begin{array}{l}\text { Proceso } \\
\text { enseñanza- aprendizaje }\end{array}$ & $\begin{array}{l}141,159,160,161,164,165,166,167,168 \\
169,173,178,179,180,181,182,183,185 \\
186,187,188,189,190,191,192,193,194 .\end{array}$ & 27 & 0.929 \\
\hline Dimensión 6 & $\begin{array}{l}\text { Inserción al contexto } \\
\text { mundial }\end{array}$ & I19, I20, I63, I70, I71, I72, I74, I75, I76, I77. & 10 & 0.846 \\
\hline
\end{tabular}

Fuente: Elaboración propia. 
Se puede manifestar que la confiabilidad de todas las dimensiones es Muy Alta; por lo tanto, se infiere que los indicadores que integran cada una de ellas muestran relación entre sí y la distribución de los ítems fue óptima, se destaca una mayor correlación en la quinta dimensión. Luego de determinar estas correlaciones, se procedió a continuar con el siguiente paso, para validar el instrumento, el cual consiste en la validez de criterio o concurrente.

\section{Validez de criterio (validez concurrente)}

Para realizar la validez de criterio se empleó el instrumento diseñado y aplicado en la Universidad de Tolima, con el fin de medir la satisfacción de los estudiantes de dicha casa de estudios. Es importante destacar que, para esta fase del estudio, el cuestionario patrón se aplicó a un grupo de 40 estudiantes que ya había sido previamente entrevistado con la otra prueba, se emplearon sus números de identificación personal para poder contrastar sus respuestas en ambos instrumentos. Vale destacar que el coeficiente del Alfa de Cronbach resultó 0,888 , el cual es muy alto, y se considera apto para continuar la investigación. Luego de haber contrastado los resultados obtenidos en ambas encuestas, se procedió a calcular el Coeficiente de Correlación de Pearson para determinar si existía relación entre ambos resultados (Tabla 9).

TABLA 9

Correlación Pearson Instrumento vs. Patrón

\begin{tabular}{|l|l|r|r|}
\hline & & PORCINST & PORCPAT \\
\hline PORCINST & Correlación de Pearson & 1 &, $706^{* *}$ \\
\hline & Sig. (bilateral) & &, 000 \\
\hline & N & 40 & 40 \\
\hline PORCPAT & Correlación de Pearson &, $706^{* *}$ & 1 \\
\hline & Sig. (bilateral) &, 000 & \\
& N & 40 & 40 \\
\hline
\end{tabular}

Fuente: Elaboración propia.

Aquí se observa un nivel Alto de correlación y se considera significativa, si se toma en cuenta que el objetivo de ambos cuestionarios era medir el nivel de satisfacción de los estudiantes universitarios con respecto a la institución; por ello se puede inferir que el instrumento cumple con el objetivo que desea medir. 
En la figura 1, se presenta el gráfico de dispersión resultante que evidencia con mayor facilidad la correlación de los resultados de la aplicación de ambos instrumentos.

FIGURA 1

Gráfico de dispersión instrumento vs. Patrón

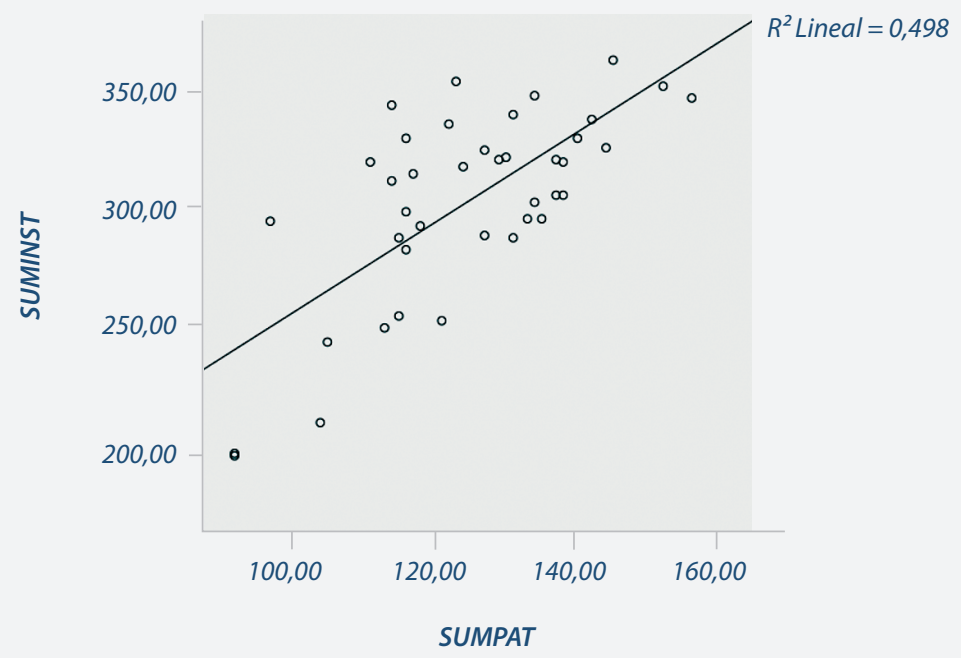

Fuente: Elaboración propia.

\section{Estabilidad del instrumento}

Para finalizar, se procedió a aplicar el instrumento en proceso de validación, a un grupo conformado por 22 sujetos previamente entrevistados con dos meses de diferencia en el tiempo, con el fin de determinar su estabilidad por repetividad y así verificar que no hubiera un sesgo por parte del investigador al momento de efectuar las encuestas y las respuestas fueran consistentes, para ello se inició con la tabulación de los resultados obtenidos en cada momento en la que fue aplicada la encuesta, para proceder a determinar la correlación entre ambos resultados se empleó el coeficiente $R$ de Pearson de nuevo; pues muestra la relación entre ambas variables, que en este caso, se refiere a la satisfacción de los estudiantes y los resultados se muestran en la Tabla 10:

TABLA 10

Correlación de Pearson para estabilidad

\begin{tabular}{|l|l|r|r|}
\hline \multicolumn{2}{|l|}{} & SESG01 & SESGO2 \\
\hline SESG01 & Correlación de Pearson & 1 &, $816^{* *}$ \\
\hline & Sig. (bilateral) & 22 &, 000 \\
\hline & N &, $816^{* *}$ & 22 \\
\hline SESG02 & Correlación de Pearson &, 000 & 1 \\
\hline & Sig. (bilateral) & 22 & 22 \\
\hline & N & 22 & \\
\hline
\end{tabular}

** La correlación es significativa al nivel 0,01 (bilateral). Fuente: Elaboración propia. 
De nuevo, se puede observar que existe una alta correlación entre las variables, lo cual indica la estabilidad del instrumento, al evidenciarse que las respuestas obtenidas en ambos momentos del tiempo fueron similares; sin embargo, para facilitar la observación de los resultados, se presenta el gráfico de dispersión obtenido en la Figura 2.

\section{FIGURA 2}

Gráfico de dispersión de estabilidad

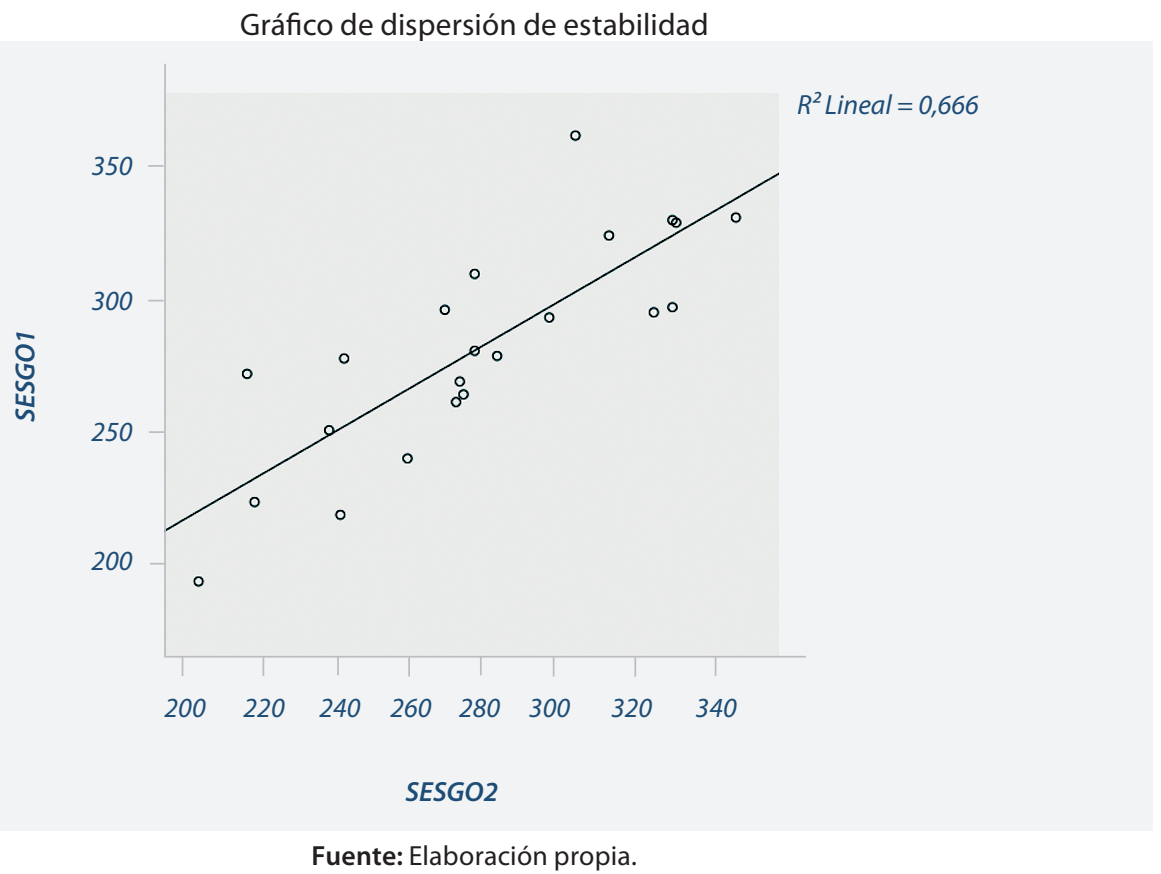

Finalmente, para comprobar los resultados anteriores, se llevó a cabo un análisis de la varianza, por medio de un modelo lineal univariante, con el fin de determinar la correlación entre las pruebas y se obtuvieron los resultados que pueden observarse en la Tabla 11.

TABLA 11

Análisis de la varianza para estabilidad Variable dependiente: SATISFACCIÓN

\begin{tabular}{|c|c|c|c|c|c|}
\hline Origen & Suma de cuadrados tipo III & GI & $\begin{array}{c}\text { Media } \\
\text { cuadrática }\end{array}$ & $\mathbf{F}$ & Sig. \\
\hline Modelo corregido & $74052,432 a$ & 43 & 1722,150 & . & . \\
\hline Intersección & 3477094,568 & 1 & 3477094,568 & . & . \\
\hline PRUEBA & 696,023 & 1 & 696,023 & . & . \\
\hline SUJETO & 66585,932 & 21 & 3170,759 & . & . \\
\hline PRUEBA * SUJETO & 6770,477 & 21 & 322,404 & . & . \\
\hline Error &, 000 & 0 & . & & \\
\hline Total & 3551147,000 & 44 & & & \\
\hline Total corregida & 74052,432 & 43 & & & \\
\hline
\end{tabular}

a. $\mathrm{R}$ cuadrado $=1,000$ (R cuadrado corregida $=$.) Fuente: Elaboración propia. 
En función de estos resultados, se puede inferir que no se evidencian mayores diferencias entre los resultados obtenidos, luego de aplicar la misma prueba dos veces a los mismos sujetos, y la mayor diferencia existente es entre las respuestas que ha dado cada uno de ellos; por lo tanto, se puede indicar que surge una correlación entre los datos obtenidos en ambos momentos del tiempo, y se infiere que el instrumento posee estabilidad.

\section{Rendimiento del instrumento. Curva COR}

Para realizar esta parte del estudio, se procedió a asignar un rango que se considera positivo y uno negativo para el análisis de los resultados de la aplicación de los instrumentos. Como esta prueba solo permite dos posibles respuestas, se procedió a generar las respuestas dicotómicas obtenidas; por lo anterior, se estableció que aquellos estudiantes que tuvieran una visión positiva de al menos 65 \% en la sumatoria de todos los aspectos en estudio, se tomarían como estudiantes satisfechos ( $\geq 303$ puntos). Luego de procesar los datos, se notó la proporción que se puede observar en la Tabla 12.

TABLA 12

Casos encontrados de estudiantes satisfechos e insatisfechos

\begin{tabular}{|l|r|r|r|c|}
\hline Clasificación & \multicolumn{2}{|c|}{ Estudiantes insatisfechos } & \multicolumn{2}{|c|}{ Estudiantes satisfechos } \\
\hline Rango instrumento & $0-302$ puntos & 16 & $303-470$ puntos & 24 \\
\hline Rango Patrón & $0-120$ puntos & 18 & $121-165$ puntos & 22 \\
\hline
\end{tabular}

Fuente: Elaboración propia.

Se puede notar que la proporción de estudiantes satisfechos e insatisfechos era similar, tanto en el instrumento en validación como en el patrón. Con estos datos dicotómicos, se procedió a realizar la curva COR, presentada en la figura 3.

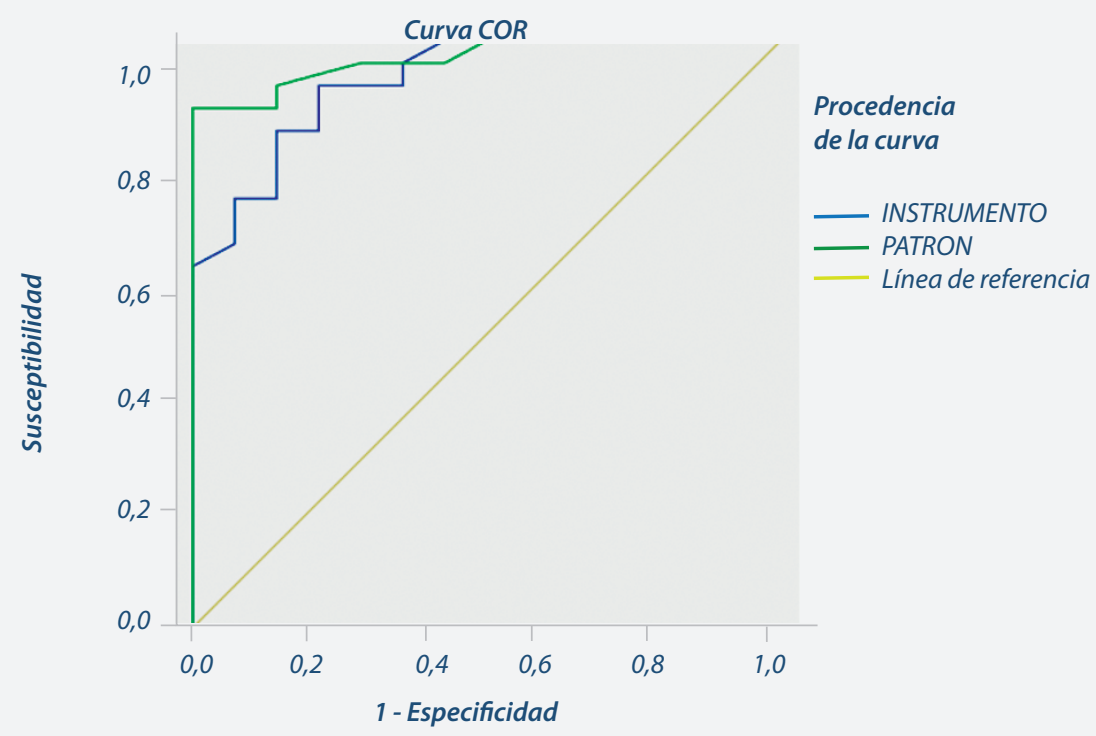

Los segmentos diagonales son producidos por los empates.

FIGURA 3 CURVA COR

Fuente: Elaboración propia 
.Para cuantificar los resultados que se observan en la figura anterior, se presenta la Tabla 13, en la cual se evidencia que el área bajo la curva del instrumento resultó ser 0,931, esto se puede traducir en que el rendimiento de la prueba es Muy Bueno. Sin duda, significa que existe una probabilidad del 93,10 \% de que la opinión de los alumnos sobre su satisfacción con la institución pueda ser medida correctamente por medio de este cuestionario

TABLA 13 Área bajo la curva COR

\begin{tabular}{|c|c|c|c|c|c|c|}
\hline & & Ár & Frrouting & áticol & $\begin{array}{l}\text { Intervalo d } \\
\text { asintótic }\end{array}$ & $\begin{array}{l}\text { nfianza } \\
95 \%\end{array}$ \\
\hline & & & & & & Límite \\
\hline & INSTRUMENTO & 931 & ,039 &, 000 & 845 & 1,000 \\
\hline Dimensioni & PATRÓN & 968 & ,023 &, 000 & 000 & 1,000 \\
\hline
\end{tabular}

a. Bajo el supuesto no paramétrico b. Hipótesis nula: área verdadera $=0,5$ Fuente: Elaboración propia.

Finalmente, para determinar el punto de corte óptimo, se procedió a calcular el Índice Youden, el cual relaciona la sensibilidad y la especificidad obtenidas, y con los resultados alcanzados se ubicó el lugar que mejor relaciona ambos elementos, se convirtió en el punto óptimo, tanto para el instrumento definitivo como para el usado como patrón, el cual indica el rango en el que los estudiantes se encuentran satisfechos e insatisfechos.

Los resultados muestran asociación con un valor positivo igual a 297,50 para el instrumento, lo cual significa que los estudiantes quienes hayan asignado al instrumento un valor total de 298 puntos o más, se encuentran satisfechos con la institución; insatisfechos se sienten aquellos que adjudican 297 puntos o menos a su cuestionario.

En el caso de la prueba patrón, el valor límite óptimo se halla en 120,5, lo cual significa que los que totalicen 121 puntos o más, se encuentran satisfechos con la institución, y quienes adjudicaron 120 puntos o menos a su cuestionario están insatisfechos, como se resume en la tabla 14 presentada a continuación:

TABLA 14 Límites de satisfacción e insatisfacción en instrumentos

\begin{tabular}{|c|c|c|c|}
\hline \multirow{2}{*}{ Cuestionario } & Condición & Valores & Dicotomizado \\
& Insatisfechos & $0-297$ & 0 \\
\cline { 2 - 4 } & Satisfechos & $298-470$ & 1 \\
\hline \multirow{2}{*}{ Patrón } & Insatisfechos & $0-120$ & 0 \\
\cline { 2 - 4 } & Satisfechos & $121-185$ & 1 \\
\hline
\end{tabular}

Con estos valores definidos, ya es posible categorizar las respuestas obtenidas luego de aplicar el instrumento a la población que desee medirse para determinar con facilidad el nivel de satisfacción estudiantil y, por otro lado, se puede utilizar para complementar la presente investigación y en futuros estudios. 


\section{SÍNTESIS Y REFLEXIONES FINALES}

Luego de haber realizado las pruebas respectivas, se determinó que el presente instrumento posee validez para medir la satisfacción de los estudiantes universitarios. Los resultados de las pruebas del Alfa de Cronbach calculados por Gento et al. (2003) y lo aplicaron en la Universidad de los Andes, resultan similares a los obtenidos luego de emplearlos en la Universidad del Zulia, lo cual podría llevar a pensar en la reproducibilidad de este; sin embargo, se debe considerar la incorporación del ítem vinculado a la iluminación de las áreas de estudio, decisión que puede ser considerada como acertada; pues no generó perturbación en la confiabilidad de la dimensión, en cambio se nota un incremento en la confiabilidad del instrumento.

Por otra parte, se pudo evidenciar que algunos de los indicadores medidos en las diez dimensiones diseñadas originalmente se podían relacionar; por lo tanto, se logró reducirlas a seis, con las cuales se puede explicar la satisfacción estudiantil y facilita los análisis posteriores. Las dimensiones finales resultaron distribuidas en condiciones físicas, servicios estudiantiles, consideración a su circunstancia económica, relaciones personales, proceso de enseñanza-aprendizaje e inserción al contexto mundial.

Adicionalmente, se observó que el instrumento diseñado por Gento et al. (2003) era uno de los más completos encontrados para medir la satisfacción estudiantil, de acuerdo con el número de indicadores que posee y la cantidad de variables que consideran que la afectan, esto incluye ítems que van desde las características físicas de la infraestructura hasta el trato recibido por parte de las autoridades; luego del análisis factorial se encuentra que la dimensión del proceso de enseñanza aprendizaje es la que muestra un nivel de correlación más alto con respecto a la satisfacción total de los estudiantes, lo cual lleva a pensar que la principal motivación que logra sentirse a gusto a los estudiantes en la institución es el hecho de ser formados en la profesión que han escogido y poder aprender a diario aspectos nuevos.

Se hace necesario destacar que el uso del presente cuestionario, permite conocer la percepción de los usuarios con respecto a su satisfacción con las características medidas; sin embargo, otras técnicas pueden utilizarse tanto para confirmar su opinión como para contrastar ambos resultados, al aplicar métodos como mediciones cuantitativas en el caso de las variables ambientales y observación directa u otros cuestionarios para entender algunos indicadores de carácter cualitativo.

Haber validado el presente instrumento, constituye solo el primer paso necesario para entender la manera en como la satisfacción estudiantil puede influenciar otras variables importantes en la formación académica del estudiantado; por lo tanto, se resulta interesante conocer las relaciones posibles con aspectos tales como: desempeño académico, calidad educativa, motivación, entre otros, dando pie a futuras investigaciones que permitan profundizar y correlacionar estos datos con variables de toda índole, con el fin de conocer los factores que pueden vincularse con el nivel de satisfacción del estudiantado, y así plantear mejoras que sean beneficiosas tanto para las instituciones, como para el alumnado.

\section{REFERENCIAS}

Albarran-Peña, José. (2019). La deserción estudiantil en la Universidad de Los Andes. Educación y Humanismo: Venezuela. 21(36).:60- 92. DOI: https://doi.org/10.17081/eduhum.21.36.2806

Baez Aumala, Mario. (2013). Factores que determinan el desempeño académico en Zamorano. Proyecto especial para optar al título de Licenciado en Ingeniería en Agro negocios. Escuela Agrícola Panamericana: Honduras. 
Basantes, René. (2017). Modelo ServQual Académico como factor de desarrollo de la calidad de los servicios educativos y su influencia en la satisfacción de los estudiantes de las carreras profesionales de la Universidad Nacional Chimborazo Riobamba - Ecuador. Tesis Doctoral. Universidad Nacional Mayor de San Marcos: Lima, Perú.

Celina H. y Campo A. (2005). Aproximación al uso del coeficiente alfa de Cronbach. Revista colombiana de psiquiatría, XXXIV(004):572-580. Asociación Colombiana de Psiquiatría: Bogotá, Colombia. Disponible en: http://redalyc.uaemex.mx/pdf/806/80634409.pdf

deVries, Wietze; León, Patricia; Romero, Joséy Hernández, Ignacio. (2011). ¿Desertores o decepcionados? Distintas causas para abandonar los estudios universitarios. Revista de la Educación Superior, México XL(4):29-49. Disponible en: https://www.redalyc.org/articulo.oa?id=60422569002.

Gento Palacios, S., Vivas García, M. (2003). El SEUE: un instrumento para conocer la satisfacción de los estudiantes universitarios con su educación. Acción Pedagógica, Venezuela. 12 (2):16-27.

González, Renato; Tinoco, Miguel y Torres Miguel. (2016). Análisis de la satisfacción de la experiencia universitaria de los egresados en 2015 de la Universidad de Colima. Paradigma Económico, México. 8(2):59-84.

Higgins, S., Hall, E., Wall, K., Woolner, P., McCaughey, C. (2005). The impact of school environments: A literature review. The center for learning and teaching. University of New Castle. S/V. Australia. S/P.

Lizzio, A., Wilson K., Simons, R. (2002) University Students' Perceptions of the Learning Environment and Academic Outcomes: implications for theory and practice. International Journal of Humanities and Social Science, India. 121:240-250. DOI: 10.1080/03075070120099359

Medina, Niorka; Fereira, Johan, et al. (2018). Factores personales que inciden en el bajo rendimiento académico de los estudiantes de Geometría. TELOS- Revista de Estudios Interdisciplinarios en Ciencias Sociales, Venezuela. 20(1):4-28. DOI: https://doi.org/10.36390/telos201.02

Potthoff, Joy. (2009). Design for communication: Post-Occupancy evaluation of classroom spaces. Open house International, United Kingdom. 34(1):26-34.

Sánchez Quintero, Jairo (2018). Satisfacción Estudiantil en Educación Superior: Validez de su medición. Universidad Sergio Arboleda. Serie Investigación, Bogotá, Colombia. 136 p.

Sandoval, María; Surdez, Edith; Domínguez, Debora. (2016). Logro Personal: factor inherente a la satisfacción estudiantil universitaria. Opción, Venezuela. 32 (13):704-724). Disponible en: https:// www.redalyc.org/articulo.oa?id=31048483034

Supo, J. (2012). Seminarios de Investigación Científica. Bioestadística. Perú.

Surdez, E. G., Sandoval, M del C. y Lamoyi, C. L. (2018). Satisfacción estudiantil en la valoración de la calidad educativa universitaria. Educación y Educadores, 21(1):9-26. doi: 10.5294/edu.2018.21.1.1 